\title{
On the Fe II Dielectronic Recombination in Stellar Envelopes
}

\author{
Alicia Cruzado ${ }^{1}$ and Adela E. Ringuelet ${ }^{2}$ \\ Facultad de Ciencias Astronómicas y Geofísicas, Universidad Nacional \\ de La Plata, Paseo del Bosque s/n, 1900-La Plata, Argentina
}

\begin{abstract}
We are interested in the atomic processes taking place in the envelopes of early type stars; the first part of our project consists of an investigation of the dielectronic recombination (DR) of metals. In earlier papers, we have studied the $\mathrm{DR}$ of $\mathrm{Mg}$ II and its contribution to the IR continuum; now, we are interested in accomplishing similar results analysing the Fe II atom.
\end{abstract}

\section{Introduction}

We started studying the $\mathrm{DR}$ of $\mathrm{Mg}$ II atoms and its significance for infrared emission of Be stars (Cruzado et al., 1998, 1999). To accomplish this, we have calculated the emissivity in the lines of $\mathrm{Mg} \mathrm{I}$ atoms after the process of electron capture has taken place. These calculations did not present serious difficulties because of the simple atomic structure of $\mathrm{Mg} \mathrm{I}$. Due to the astrophysical significance of $\mathrm{Fe}$ II, we would like to investigate the DR of this element. However, the complex structure of the Fe I atom does not allow us to deal with this problem as we did with $\mathrm{Mg}$ I. The great number of emission lines originated in the electronic transitions among different energy levels of the Fe I atom, does not permit us to consider them individually. Therefore, in order to estimate the influence on the infrared continuum of Be stars of the DR of Fe II atoms, we have adopted a statistical method based upon the distribution with wavelength of the number of lines and the value of the transition probabilities,

\section{Discussion}

In studying the $\mathrm{DR}$ of $\mathrm{Mg}$ II atoms we have arrived at the following expression for the emissivity in the line with wavelength $\lambda_{l h}$

$$
\epsilon\left(\lambda_{l h}\right)=N_{e} N(\mathrm{Mg} \text { II }) \frac{P_{l h}^{R}}{\sum_{k<l} P_{l k}^{R}} \sum_{j}\left[\frac{g_{j}}{2 g_{m}}\left(\frac{h^{2}}{2 \pi m K T}\right)^{\frac{3}{2}} e^{\frac{-E_{j}}{K T}} P_{j h}^{R}\right] h \nu_{l h}
$$

\footnotetext{
${ }^{1}$ Fellow of the University of La Plata

${ }^{1}$ Member of the Carrera del Investigador Científico, CONICET, Argentina
} 
with:

$P_{l h}^{R}$ : the radiative probability for the $l \longrightarrow h$ transition $\left[\mathrm{s}^{-1}\right.$,

$l, k, h$ : bound states of a $\mathrm{Mg} \mathrm{I}$ atom,

$j$ : autoionizing state of a $\mathrm{Mg} \mathrm{I}$ atom,

and $E_{j}$ : autoionizing state energy over the ionization limit.

The remaining symbols have their usual meaning.

Then, we have added the emissivity in all the lines with $\lambda_{1} \leq \lambda \leq \lambda_{2}$, where $\lambda_{1}$ and $\lambda_{2}$ define the wavelength ranges of the Johnson system filters.

The significance of this process in the stellar flux, may be inferred by computing

$$
4 \pi^{2} R_{*}^{2} \int_{\lambda_{1}}^{\lambda_{2}} B_{\lambda} d \lambda+\frac{4}{3} \pi\left(D^{3}-d^{3}\right) \sum_{\lambda_{1}}^{\lambda_{2}} \epsilon\left(\lambda_{l h}\right)
$$

where $B_{\lambda}$ is Planck's function and $D$ and $d$ are the outer and inner radii of the region where $\mathrm{DR}$ occurs (we have considered this region to be a homogeneous transparent region, located far away from the star, with constant temperature and constant density).

In this case, all the atomic parameters were obtained applying the HartreeFock methodology, by using Cowan's code.

At present, instead of dealing with the atomic parameters individually, we are trying to find out their general behavior. First, we accomplished this with the $\mathrm{Mg}$ I parameters to compare the results obtained in this way with earlier results. Then, working with the Fe I atom, we aim at reaching some conclusion about the significance of DR of Fe I atoms on the emitted flux of the Be stars.

\section{Results and Conclusions}

In the case of Fe I, IR excesses will be present simultaneously with very large UV excesses. Since those huge UV excesses are not observed in Be stars, we can infer that the DR of Fe II is negligible in the IR.

The conclusion is that DR of Fe II may contribute to the UV excess but very improbably to an IR excess.

\section{References}

Cruzado, A., Di Rocco, H.O., Ringuelet A. 1998, ApJ 503, 902-915 (Paper I). Cruzado, A., Ringuelet, A. 2000, this Volume. 